\title{
Influence of Autonomic Control on the Specific Intermittent Performance of Judo Athletes
}

\author{
by \\ Bruno T. Campos ${ }^{1}$, Eduardo M. Penna ${ }^{1,2}$, João G.S. Rodrigues ${ }^{1}$, Mateus Diniz', \\ Thiago T. Mendes ${ }^{1,3}$, André F.C. Filho ${ }^{4}$,Emerson Franchini ${ }^{5}$, Fabio Y. Nakamura6,7, \\ Luciano S. Prado ${ }^{1}$
}

Judo is a high-intensity intermittent combat sport which causes cardiac adaptations both morphologically and related to the autonomic nervous system (ANS). Therefore, this study aims to verify the correlation between heart rate variability (HRV) at rest with performance in the Special Judo Fitness Test (SJFT) and whether groups with different $R R$ values at rest show different performance in the SJFT and during post-test recovery. Sixteen judo athletes with 7.2 \pm 3.9 years of training experience participated in the study. Before and after the SJFT execution HRV and lactate measurements were conducted. For HRV analysis, we used the mean interval $R R$, the standard deviation of the RR interval (SDNN), the root mean square of successive differences in RR intervals (RMSSD), the low frequency (LF) and high frequency (HF) in normalized and absolute units. The sample was split into two groups (low RR and high RR) to verify if this variable could differentiate between specific performance. For the SDNN, a significant and moderate correlation $(r=0.53)$ was found with the total number of throws and throws in the series $A(r=0.56)$ and $B(r=0.54)$ and for the RMSSD a correlation with throws during series $B(r=0.59)$ in the SJFT. However, the groups did not differ in performance and recovery. Therefore, HRV is related to intermittent judo performance; however, it cannot differentiate between judokas at different levels of performance.

Key words: heart rate variability, anaerobic performance, Special Judo Fitness Test.

\section{Introduction}

Judo is a high-intensity and complex intermittent combat sport. It has a high demand for technical and tactical skills and requires precision, strength, and speed (power) during the execution of throwing techniques, which must be performed with perfect timing so that the athlete achieves higher competitive performance (Franchini et al., 2011a). In international judo competitions, elite athletes perform between 5 to 7 fights interspersed by intervals of 10 to $15 \mathrm{~min}$.
This makes it necessary to simultaneously develop aerobic power and capacity for better recovery between each match (Detanico et al., 2012).

Cardiac morphological adaptations have been associated with judo practice (Laskowski et al., 2008) as well as greater rest vagal modulation when compared to sedentary subjects (Araújo et al., 2016). Vagal modulation can be evaluated through heart rate variability (HRV), which

\footnotetext{
1 - Universidade Federal de Minas Gerais, Brasil.

2 - Universidade Federal do Pará - Castanhal Campus, Brasil.

3 - Universidade Federal do Maranhão - Pinheiro Campus, Brasil.

4 - Centro de Treinamento Esportivo, Universidade Federal de Minas Gerais, Brasil.

5 - Universidade de São Paulo, Brasil.

6 - The College of Healthcare Sciences, James Cook University, Queensland, Australia.

7 - Department of Medicine and Aging Sciences, "G. d'Annunzio" University of Chieti-Pescara, Italy.
} 
mathematically describes oscillations of the intervals between consecutive heart beats (RR intervals). It depends on the autonomic nervous system (ANS) control over the sinus node. HRV can identify differences in the SNA control in healthy individuals, athletes, and diseased patients (Mikołajec et al., 2017; Aubert et al., 2003; Task Force, 1996). In sport, resting HRV has been related to cardiorespiratory fitness and weekly training loads ( Mikołajec et al., 2017; Buchheit and Gindre, 2006).

In judo, resting HRV has been used as a physiological indicator of pre-competitive anxiety (Morales et al., 2012) and as a monitoring tool to determine the effects of different training loads (Morales et al., 2014; Vacher et al., 2016). In addition, Blasco-Lafarga et al. (2013) found a relationship between higher resting vagal modulation and better supramaximal performance in the Blasco-specific judo test (BSJT). However, while the results found by Blasco-Lafarga et al. (2013) indicate the relationship between HRV and performance in a judo test, no study has yet investigated the relationship between HRV and the level of performance of judo athletes in tests more specifically designed for the modality or as a criterion of their physical fitness level. Thus, when comparing groups with different HRV, it would be possible to infer the specific and individual athlete's fitness level to judo practice.

To determine the specific fitness level to judo practice, the Special Judo Fitness Test (SJFT) has been proposed, and this test can determine the level of aerobic-anaerobic fitness via an index calculated from the total number of throws and the heart rate measurements after the test (Sterkowicz et al., 1999). It seems to be sensitive and able to differentiate between elite and nonelite judo athletes (Franchini et al., 2005, 2007). This test involves judo-specific throwing technique executions during a given time, which resembles the effort-pause ratio of the match. The physiological demand of the test is similar to that observed during judo matches (Franchini et al., 2011a, 2011b), and the results have a positive and significant correlation with aerobic and anaerobic fitness indexes (Sterkowicz et al., 1999). The SJFT results can determine the level of aerobicanaerobic fitness of the athletes considering a reference table that classifies the performance
(Franchini et al., 2009).

Furthermore, the SJFT can distinguish between athletes at different levels of performance (Franchini et al., 2009), and correlates with cardiorespiratory (Garbouj et al., 2016) and neuromuscular fitness levels (Boguszewska et al., 2010). HRV also relates to training status (Hedelin et al., 2001), and it is hypothesized that HRV indices could indicate the performance level of judo athletes while using a valid test for the modality.

Athletes and active individuals with greater vagal resting modulation usually show faster recovery of vagal tone and blood lactate removal (Blasco-Lafarga et al., 2013; Molina et al., 2016). This may be an important characteristic in judo as it involves many sequential matches, and accelerated recovery may be a differential for a good performance in subsequent matches (Franchini et al., 2009).

However, no study has yet evaluated athletes with different resting vagal modulation (high and low RR intervals). This division, based on a previous study (Hansen et al., 2003), uses the median RR interval to classify individuals. Thus, this study aims to verify the correlation of HRV at rest with the performance in the SJFT and if groups with different $R R$ values at rest present different performance in the SJFT and in post-test recovery. We hypothesized that resting HRV would correlate with performance and that the higher RR group would present superior performance in the SJFT and faster recovery than the lower RR group.

\section{Methods}

\section{Participants}

Sixteen judo athletes of both sexes (12 men and 4 women) performing at a regional competitive level participated in this study. The athletes displayed age of $19.6 \pm 2.9$ years, body mass of $67.9 \pm 12.1 \mathrm{~kg}$ (distributed in 4 weight categories: $3-60 \mathrm{~kg}, 5-66 \mathrm{~kg}, 7-73 \mathrm{~kg}, 1+100 \mathrm{~kg}$ ), body height of $168.5 \pm 5.1 \mathrm{~cm}$, fat mass of $11.49 \pm$ $3.90 \%$ and had at least 3 years of experience in the sport $(7.2 \pm 3.9$ years, and $11.2 \pm 4.6$ practice hours per week). Athletes who competed in the State Championship in the year of the study were included. They were training regularly and had no recent musculoskeletal injuries. The procedures were performed in accordance with 
the ethical standards of the Declaration of Helsinki. The participants signed an informed consent form, and all procedures were approved by the local university Research Ethics Committee (protocol number: 55286716.0.0000.5149).

\section{Measures}

Initially, the volunteers underwent anthropometric evaluation and then remained in a seated position for 10 minutes to record the RR intervals. Next, 30 microliters of blood were collected from the earlobe to measure blood lactate concentration. The volunteers then completed the SJFT (they were already familiar with the test). Blood samples were collected immediately after and 3, 5 and 10 minutes after the test for the measurement of blood lactate concentration. Concomitantly, HRV was recorded for $10 \mathrm{~min}$ after the end of the test. All procedures were performed between 8:00 and 10:00 am, and the volunteers were asked not to drink alcohol or caffeine or exercise during the last 24 hours prior to testing.

Anthropometry:

Body mass $(\mathrm{kg})$ was measured barefoot, with volunteers wearing only shorts using a digital scale; and body height $(\mathrm{cm})$ was measured using a stadiometer with accuracy of $0.5 \mathrm{~cm}$ (Filizola ${ }^{\circledR}$ ). The skinfold thicknesses (subscapular, triceps, biceps, pectoral, subaxillary, suprailiac, abdominal, thigh, and calf) were measured with a plicometer (Lange ${ }^{\circledR}$ ) according to the protocol proposed in the literature (Jackson and Pollock, 1978).

Special Judo Fitness Test:

The SJFT is used to evaluate athletes' specific fitness. This test consists of two Ukes (athletes who will be projected) with body mass similar to the performer separated by 6 meters and a Tori (an athlete who will execute the projections) separated by 3 meters from each Uke (Figure 2). After a sound signal, the Tori must run towards Uke and execute the ippon-seoi-nage technique and immediately run towards the other Uke executing the same technique. The Tori was instructed to execute as many repetitions as possible in a given time.

The test is divided into three series (Throwsa, ThrowsB and Throwsc) with a recovery period of $10 \mathrm{~s}$ between sets. The first series (A) lasts $15 \mathrm{~s}$, and the other two (B, C) last $30 \mathrm{~s}$ each. The heart rate (HR) was recorded immediately at the end (HRPost) of the test and one minute after

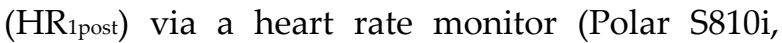
Finland). To determine the performance in the SJFT, the Performance Index (ISJFT) was calculated according to a specific equation for this test by summing the final $\mathrm{HR}_{\text {post }}$ and $\mathrm{HR}_{1 \text { post, }}$ and the resulting value was divided by the sum of the total number of throws (Throwstotal), i.e., (IsjFT $=$ $\left[\mathrm{HR}_{\text {post }}+\mathrm{HR}\right.$ 1post $_{\text {/ }} /$ Throwstotal). In this test, the lower the IsfFT, the higher the performance (Franchini et al., 2009).

Heart Rate Variability:

The HRV measurements were performed before and after the SJFT. The volunteers remained seated and were instructed to remain calm and quiet with spontaneous breathing and with minimal body movement. A cardiofrequencimeter (Polar ${ }^{\circledR}$ RS810i, Finland) was used for data acquisition. Data were exported to Polar Precision Performance Software (Polar Electro $\mathrm{Oy}$ ) and $\mathrm{RR}$ ranges were exported in ASCII format for further analysis. The RR intervals were analyzed in Kubios HRV analysis software, version 2.0 (Biosignal Analysis and Medical Imaging Group - University of Kuopio, Kuopio, Finland). All data were visually inspected to remove ectopic beats and, when identified, the "low" filter of Kubios software was used (the analyzed sections had no more than $3 \%$ of the removed section) (Tarvainen et al., 2014). Those manually removed sections were replaced by interpolation of the adjacent RR intervals (Task Force, 1996). Following previous methods (Nunan et al., 2006; Panissa et al., 2016), the last 5 minutes of analysis were used.

The HRV variables analyzed in the time domain were the mean RR interval (Mean RR), the standard deviation of the RR interval (SDNN), and the square root of the sum of the squares of the adjacent RR intervals (RMSSD). To analyze the frequency domain, the fast Fourier transform (FFT) of the RR signals was performed. The spectral response provided by the system was divided into 2 bands: low frequency - LF (0.04$0.15 \mathrm{~Hz})$ and high frequency - HF $(0.15-0.4 \mathrm{~Hz})$. The LF and HF data were presented from their natural logarithm (LnLF, LnHF) of absolute power $\left(\mathrm{ms}^{2}\right)$ and in normalized units (u.n.) from which the influence of the VLF band was excluded (Task Force, 1996).

The HRV recording after the SJFT was 
divided into five 2-min sections for analysis of the HRV recovery curve. For this short-term analysis, only the natural logarithm of the RMSSD (LnRMSSD) was used based on a previous study (Buchheit et al., 2007). Prior to this analysis, the two groups were separated through the median values of the RR interval at rest: the high $R R$ group and the low RR group (Hansen et al., 2003). The LnRMSSD recovery values were analyzed in these groups.

Blood lactate concentration:

Blood samples $(30 \mu \mathrm{L})$ were collected from the earlobe using disposable lancets and heparinized capillaries. Samples were stored in tubes containing $60 \mu \mathrm{L}$ of $1 \%$ sodium fluoride $(\mathrm{NaF})$ and frozen at $-80^{\circ} \mathrm{C}$ for further evaluation. Blood lactate concentration was determined in duplicate via an electroenzymatic method (YSI 1500 SPORT, Yellow Springs, OH, USA). Samples were collected before exercise and immediately after as well as 3, 5 and $10 \mathrm{~min}$ after exercise. From these samples, the highest lactate concentration was denominated as [LAC] $]_{\text {peak. }}$

\section{Statistical analysis}

Data normality, homoscedasticity, and sphericity were confirmed before the inferential analyzes by the Shapiro-Wilk, Levene and Greenhouse-Geiser tests, respectively. When the variable was not normally distributed, it was transformed into a natural logarithm. For inferential analyses, a significance level of $p \leq 0.05$ was used. All data are described as the mean \pm standard deviation. The Pearson's correlation coefficient was used to test the correlation between resting HRV and physical performance. The magnitude of the correlation was classified from the value of $r$ with values $<0.3$ considered small, 0.3 - 0.5 moderate, $0.5-0.7$ high, and $>0.7$ very high (Cohen, 1988).

To compare different groups of resting HRV, groups were separated by the median values of the $R R$ interval including the high $R R$ group (HRR) and the low RR group (LRR). HRV indexes were described for the two groups, and an independent $t$-test was used to verify the differences between the means of the groups in HRV, the SJFT, and [LAC]peak. For analysis of HRV and the lactate recovery curve after the SJFT, a two-way ANOVA was used (group $\times$ time), with repeated measures on time factor. When differences between the means were detected, the
Bonferroni post-hoc test was used to identify the differences. The effect size was calculated using Cohen's $d$ for comparisons between groups. The $d$ values up to 0.20 were considered small; 0.50 was a medium effect and over 0.80 large (Cohen, 1988). All data were analyzed using the statistical package SigmaPlot 11.0 (Systat Software Inc, USA).

\section{Results}

The RR-intervals were different between groups (HRR and LRR) in the time domain ( $p<$ $0.05)$, but not in the frequency domain (Table 1). Furthermore, performance between HRR and LRR groups in the SJFT and [LAC]peak was not different.

The analysis of the LnRMSSD performed during recovery (Figure 2) did not find an

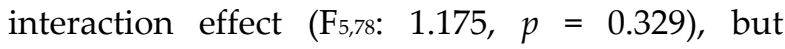
identified a significant effect for the group factor $\left(F_{1,78:} 10.788, p=0.002\right)$, with higher LnRMSSD values in the HRR when compared to the LRR group. A significant time effect was found ( $\mathrm{F}_{5-78:}$ 16.874, $p<0.001$ ). All time points after the SJFT had lower values of LnRMSSD, when compared to Pre $(p<0.001)$. When comparing the values between the groups, small-to-large effect size values were found throughout recovery (Post 1: 0.41; Post 2: 0.67, Post 3: 0.77, Post 4: 0.77, and Post 5: 1.4).

Blood lactate concentration [Lac] did not

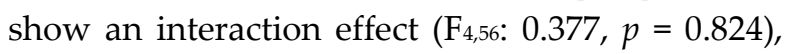
but presented a time main effect $\left(\mathrm{F}_{4,56: 50.242, p<}\right.$ 0.001). [Lac] was higher in the post-test compared to the pre-test moment. After the SJFT, Post 3 and 4 were higher ( $p<0.001$ for both comparisons) than Post 1 and 2. When comparing the values between the groups, small to moderate effect size values were found throughout recovery (Post 1: 0.52; Post 2: 0.29, Post 3: 0.07, and Post 4: 0.18).

Regarding HRV rest values, only the SDNN variable (a global index representing the sympathetic and parasympathetic nervous system) showed a significant and moderate correlation $(\mathrm{r}=0.53 ; p<0.05)$ with Throwstotal (Figure 3). When the relationship between resting HRV and the different SJFT series was established, the SDNN index showed a positive and moderate correlation with the number of throws in the A series (ThrowsA) $(r=0.56, p<0.05)$ 
and B series (ThrowsB) $(\mathrm{r}=0.54, p<0.05)$. The RMSSD index correlated only with performance in the B series $(r=0.59, p<0.01)$ (Figure 4). In addition, the LnHF index correlated positively and strongly with the $\mathrm{HR}_{\text {post }}(\mathrm{r}=0.55, p<0.05)$.
There was no significant correlation between the HRV indices (Mean RR, SDNN, RMSSD, LFnu, HFnu, LnHF, and LnLF) and the number of throws in the $\mathrm{C}$ series (Throwsc), HR1post, IsfFr, and [LAC $]_{\text {peak. }}$

Table 1

Comparison of heart rate variability indexes between groups of low and high $R R$

\begin{tabular}{ccccc}
\hline & \multicolumn{4}{c}{ HRV } \\
\cline { 2 - 5 } Index HRV & $\begin{array}{c}\text { Low RR } \\
(\mathrm{n}: 8)\end{array}$ & $\begin{array}{c}\text { High RR } \\
(\mathrm{n}: 8)\end{array}$ & $p$ & Cohen's $d$ \\
\hline Mean RR (ms) & $719.7 \pm 74.2$ & $976.6 \pm 100^{*}$ & $<0.001$ & 2.91 \\
SDNN (ms) & $73.3 \pm 23.5$ & $124.5 \pm 27.7^{*}$ & 0.001 & 1.99 \\
RMSSD (ms) & $35.2 \pm 12.3$ & $74.7 \pm 27.9^{*}$ & 0.003 & 1.83 \\
LF (n.u.) & $64.4 \pm 21.3$ & $60.3 \pm 24.8$ & 0.72 & 0.17 \\
HF (n.u.) & $35.4 \pm 21.3$ & $39.6 \pm 24.8$ & 0.72 & 0.18 \\
LF/HF & $2.6 \pm 2.0$ & $2.9 \pm 3.0$ & 0.84 & 0.11 \\
Ln LF (m.s $\left.{ }^{2}\right)$ & $-2.5 \pm 0.3$ & $-2.5 \pm 0.2$ & 0.80 & 0.00 \\
Ln HF (m.s s $\left.^{2}\right)$ & $-1.6 \pm 0.1$ & $-1.4 \pm 0.3$ & 0.08 & -0.89 \\
\hline
\end{tabular}

Mean interval $R R$ (Mean $R R$ ), Standard deviation intervals $R R(S D N N)$, root means square of successive differences in the RR intervals (RMSSD), LnHF = natural logarithm high frequency;

$L n L F=$ natural logarithm low frequency $H F=$ high frequency; $L F=$ low-frequency;

$L F / H F=$ low-to-high frequency ratio, Low $R-R$ and High $R-R=$ groups divided according to the median $R R$ interval. * ${ }^{*}$ ignificant difference $(p<0.05)$.

Table 2

Comparison of performance in the SJFT between groups of low and high RR Special Judo Fitness Test High RR (n:8) Low RR (n:8) Cohen's

\begin{tabular}{ccccc}
\hline Throwstotal & $26.3 \pm 1.4$ & $26.0 \pm 2.9$ & 0.74 & 0.13 \\
IsjFT & $11.68 \pm 0.74$ & $11.67 \pm 1.37$ & 0.99 & 0.00 \\
HR $_{\text {post }}\left(\mathrm{b} \cdot \mathrm{min}^{-1}\right)$ & $168 \pm 7$ & $163 \pm 20$ & 0.46 & 0.33 \\
HR $_{\text {1post }}\left(\mathrm{b} \cdot \mathrm{min}^{-1}\right)$ & $139 \pm 9$ & $140 \pm 19$ & 0.96 & 0.06 \\
Lactate Peak $(\mathrm{mmol} / \mathrm{L})$ & $14.26 \pm 3.36$ & $13.95 \pm 3.31$ & 0.85 & 0.09
\end{tabular}

Throwstotal $=$ set $A+\operatorname{set} B+\operatorname{set} C ;$ ISJFT $=$ sum of the HR after and HR 1 min after divided by the total number of projections; $H R_{\text {post }}=H R$ at the end of the test; $H R_{1 p o s t}=H R 1$ min after the test; Low $R-R$ and High $R-R=$ groups divided according to the median $R R$ interval. 


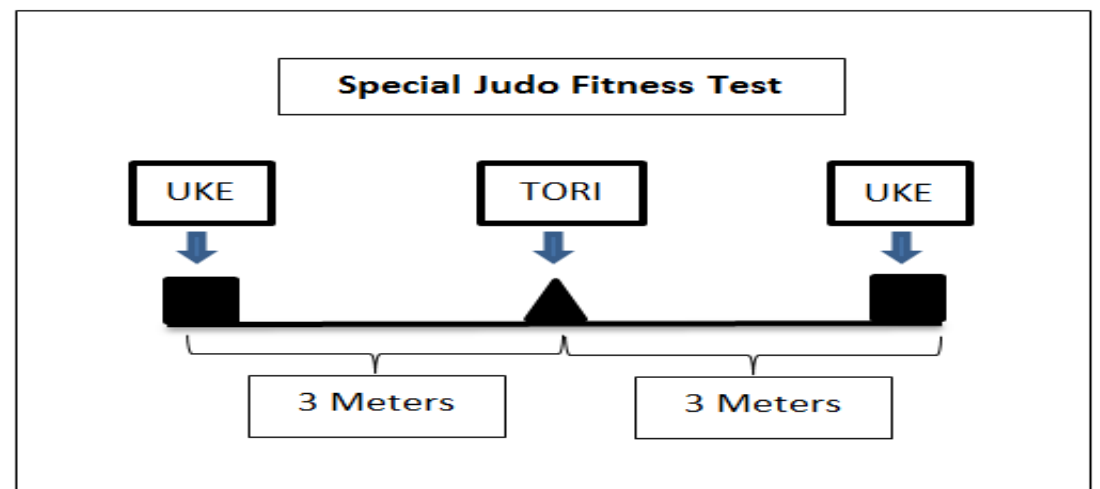

Figure 1

Special Judo Fitness Test

Special Judo Fitness Test consists of two Ukes (athletes who will be projected) separated by 6 meters and a Tori (an athlete who will execute the projections)

separated by 3 meters from each Uke.
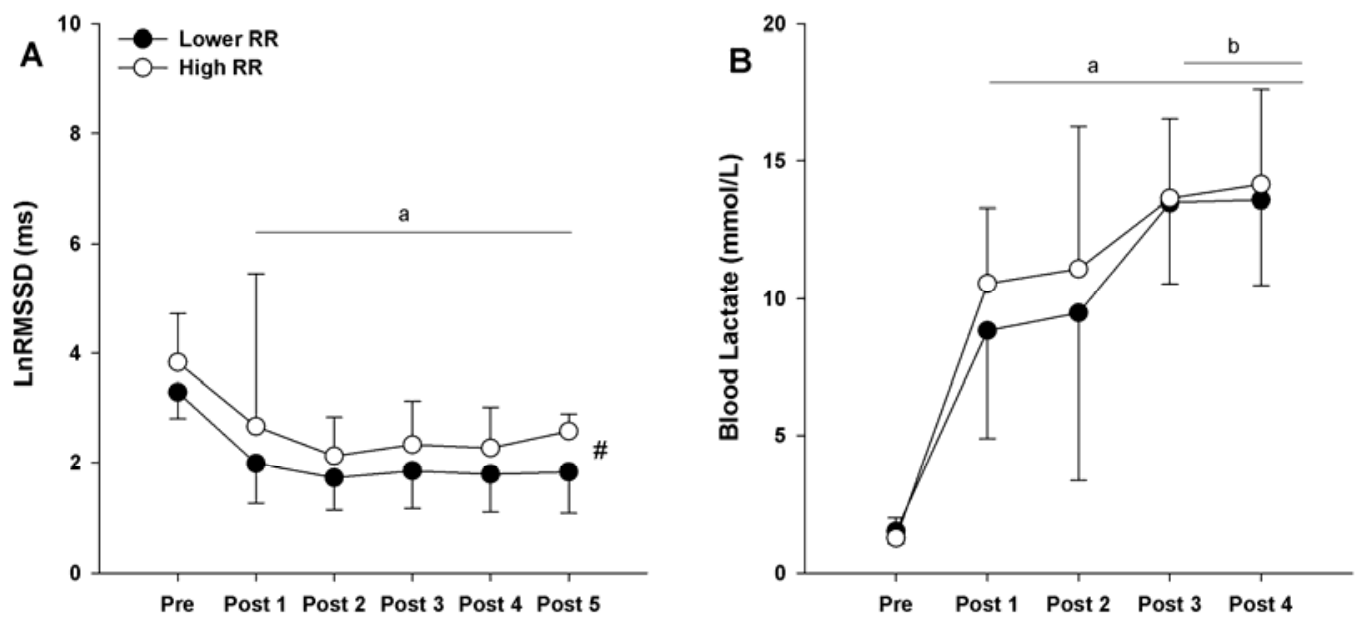

Figure 2

LnRMSSD and Blood Lactate Recovery

Index LnRMSSD (natural logarithm root means square of successive differences in the RR intervals)

(A) and Blood Lactate (B), before (Pre), immediately after up to 2 min post (Post 1),

from the $2^{\text {nd }}$ until the $4^{\text {th }}$ min (Post 2), from the $4^{\text {th }}$ until the $6^{\text {th }}$ min (Post 3 ),

from the $6^{\text {th }}$ until the $8^{\text {th }}$ min (Post 4), from the $8^{\text {th }}$ until the $10^{\text {th }}$ min (Post 5)

of the SJFT performance. \# Main group effect $(p<0.05)$;

$a=$ Significant difference $(p<0.05)$ in relation to the pre;

$b=$ Significant difference $(p<0.05)$ in relation to the Post 1 and 2 . 

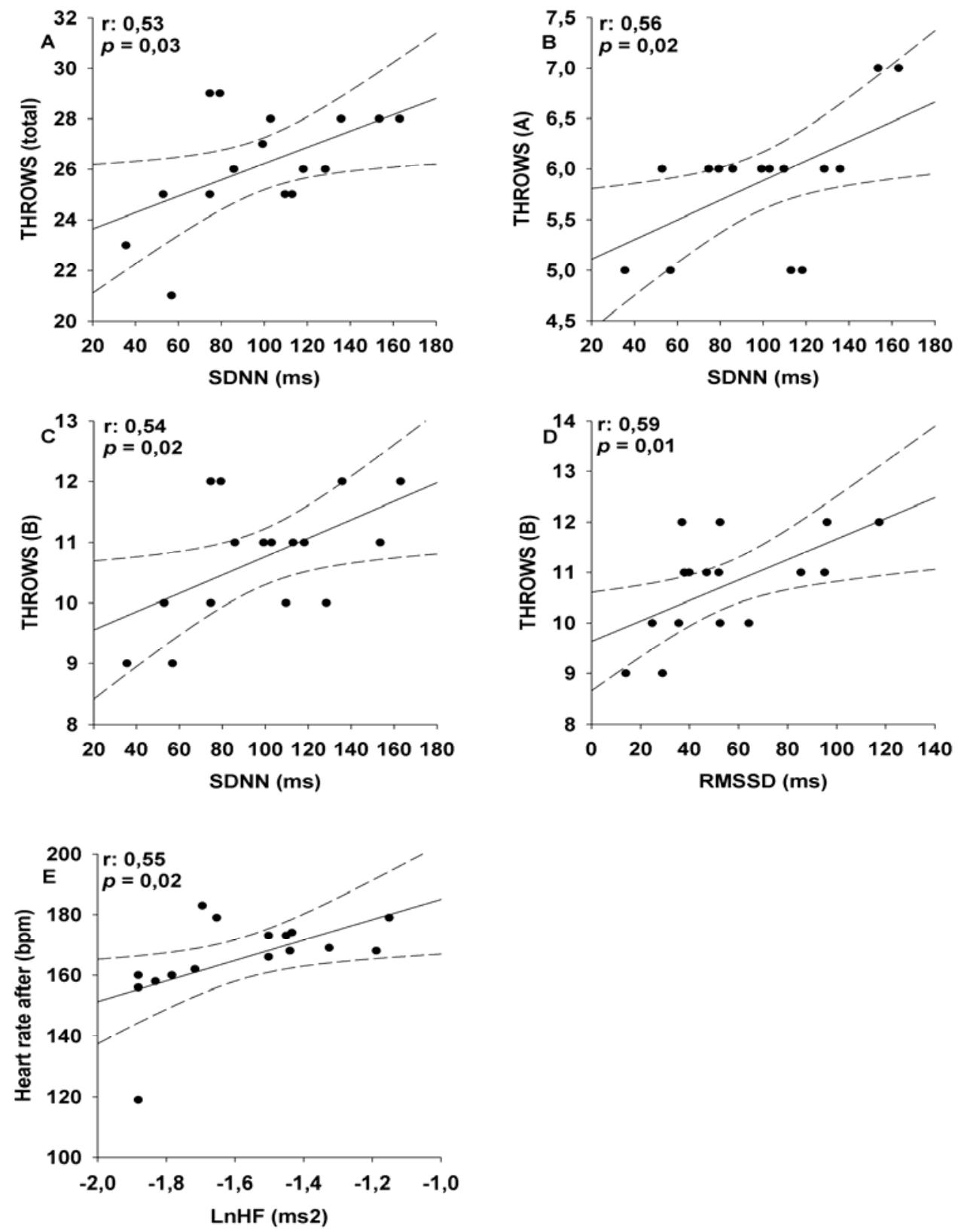

Figure 3

Correlation between HRV indexes SJFT performance

Correlations between HRV (x-axis) indices and SJFT variables (Total throws [THROWS total, throws in series $A\left[T H R O W S_{A}\right]$ and $B$ [THROWS $B$ and HR immediately at the end of the test- $y$-axis). Standard deviation intervals $R R(S D N N)$, root means square of successive differences in the RR intervals (RMSSD), LnHF = natural logarithm high frequency. Dashed line: 95\% confidence interval. Significant difference $(p<0.05)$. 


\section{Discussion}

The aim of this study was to determine if resting HRV could classify athletes at different levels of performance in the SJFT and to verify if the athletes who presented greater resting bradycardia had more accelerated recovery of the vagal activity and blood lactate concentration after exercise than the group with a low RR rest interval. When comparing the groups (HRR and LRR), no significant differences in test performance and lactate responses during recovery were observed. However, the HRR group presented higher LnRMSSD values throughout the 5-min recovery after the SJFT. Variables related to the time domain (SDNN and RMSSD) showed moderate correlation with Throwstotal, ThrowsA, and Throwsв. Furthermore, the LnHF correlated with the HR post obtained in the test. However, no HRV variable correlated with IsJFT, HR1post or [Lac]peak. Hence, the hypothesis that the resting vagal modulation would influence the intermittent performance was not confirmed. HRV could not classify the athletes according to their performance in the SJFT. However, a moderate relationship between performance and the baseline level of HRV could be observed.

Moderate correlations between Throwstotal (SDNN), Throwsa (SDNN) and Throwsв (SDNN; RMSSD) were observed, indicating the relationship between performance and vagal modulation indices. Thus, the level of parasympathetic activity at rest may influence the performance evidenced by the total number of throws and throws in each series. The results demonstrate the relationship between vagal resting modulation and anaerobic performance, confirming results of Blasco-Lafarga et al. (2013), who also found a significant correlation ( $\mathrm{r}: 0.51, p$ $<0.05$ ) between resting RMSSD and the final BSJT score. However, the test used in the present study had shorter duration when compared to the one utilized by Blasco-Lafarga et al. (2013), which might result in a greater anaerobic contribution.

Although the relationship between resting HRV and the number of throws shows a possible association between HRV and anaerobic performance, no correlation between HRV and [LAC] $]_{\text {peak }}$ (understood in previous studies as an indicator of glycolytic pathway solicitation) was observed. However, the relationship between
HRV and anaerobic energy pathway recruitment seems to exist as shown by authors who found a significant correlation between anaerobic performance and resting HRV (Cataldo et al., 2016).

The LnHF variable, which also represents the vagal activity on the sinoatrial node, was correlated with $\mathrm{HR}_{\text {post }}(\mathrm{r}=0.55, p=0.02)$, in accordance to the results found by Blasco-Lafarga et al. (2013). It is proposed that the upper parasympathetic modulation aids in the peak HR response allowing a rapid parasympathetic withdrawal at the beginning of the exercise in conjunction with a maximal sympathetic impulse making it possible to reach a higher peak HR (Douglas et al., 2016; Hynynen et al., 2008). Therefore, greater resting vagal modulation would provide greater sympathetic discharge and maximum cardiac performance.

The groups were significantly different in the time domain variables (RMSSD and SDNN) indicating that the HRR group had a higher vagal tone. However, the frequency domain did not differentiate between the groups. Some authors argue that all HRV variables represent vagal control, which does not imply that they provide the same information regarding the autonomic regulation (Reyes et al., 2013). Here, only the time domain variables were related to performance, while the variables in the frequency domain were not. When the groups were separated, the frequency domain was not different, thus the homogeneity of the groups in the frequency domain may explain lack of the correlation with the performance.

Despite the relationship between HRV at rest and SJFT performance, we could not classify judo athletes in performance levels via the vagal characteristics of the athletes - there was no correlation with the ISJFT or differences in performance when compared to the groups. This may be related to the homogeneity of the sample used, which included only regional-level judo athletes. In fact, the two groups presented a "regular" result in the total number of throws and "excellent" in the HR values immediately after the test, 1 min after the test and in the SJFT index, when classified according to reference values of this test (Franchini et al., 2009). Thus, the homogeneity of the groups in SJFT performance may have limited the identification of significant 
correlations.

When the vagal return after the SJFT was analyzed, there was a group main effect in which the group with higher previous vagal indexes also showed higher recovery values. High modulation of cardiac vagal tone post-exercise is associated with high basal vagal cardiac tone and good aerobic fitness (Tulppo et al., 2011). However, LnRMSSD values remained lower than preexercise values for both groups. This reduction in post-exercise vagal tone may be related to the high sympathetic activity that occurs during exercise and the persistent elevation of adrenergic factors and local metabolites during recovery (Buchheit et al., 2007). Lower values of LnRMSSD after exercise may be related to blood lactate response, which peaked around the $10^{\text {th }} \mathrm{min}$ demonstrating a high local metabolite concentration during recovery, which could have prevented the vagal indices from returning to resting levels.

Judo performance depends on high energy transfer rates (Franchini et al., 2013). Here, vagal modulation showed a significant correlation with performance (which depends on successive explosive actions). HRV can be an important tool for performance control. Morales et al. (2014) demonstrated that HRV could be used as a tool to control training loads; thus, this variable allows the daily control of the individual's response to the training load and the predisposition for highintensity performance. However, this hypothesis needs to be tested by adopting longitudinal study designs in which the same subject is evaluated (SJFT) on days when the subject has higher and lower HRV values at rest.

Although a relationship between performance and the autonomic modulation in resting judo athletes was observed, it was not possible to use this tool as a criterion for the classification of the athletes' fitness level. Thus, a possible limitation of the study may be the sample size, which did not allow a greater stratification of the subjects in the performance groups via reference values (Franchini et al., 2009), making it impossible to compare HRV in different SJFT classifications. In addition, another limitation of the study was that the athletes' menstrual cycle stage was not recorded, since different phases may influence HRV (Bai et al., 2009; Brar et al., 2015) and help explain the results. In future experiments, it will be interesting to verify the correlations between HRV and judo performance in three distinct groups (low, medium and high HRV). For this purpose, a larger number of athletes should be tested.

\section{Conclusion}

The rates of vagal tone in the time domain of resting HRV correlated positively with specific intermittent performance of judo athletes evidenced by the number of throws. In addition, individuals with higher RR interval values at rest presented higher LnRMSSD values at recovery. However, HRV could not classify athletes according to their level of performance in the SJFT. Thus, in spite of the relationship between HRV and judo performance, more studies with larger samples are needed to verify whether this variable can classify athletes with different levels of performance, since the correlation analyzes cannot be understood as a cause and effect relation.

\section{References}

Araújo PS, Carvalho WRG, Navarro F, Rodrigues B, Gambassi BB, Ramallo, BT, Mostarda, CT. Cardiac autonomic modulation in judo athletes : evaluation by linear and non-linear method. Sport Sci Health, 2016; 12: $125-130$

Aubert A, Seps B, Beckers F. Heart rate variability in athletes. Sports Med, 2003; 33: 889-919. https://doi.org/10.2165/00007256-200333120-00003

Bai X, Li J, Zhou L, Li X. Influence of the menstrual cycle on nonlinear properties of heart rate variability in young women. Am J Physiol Heart Circ Physiol, 2009; 297: 765-74

Blasco-Lafarga C, Martínez-Navarro I, Mateo-March, M. Is Baseline Cardiac Autonomic Modulation Related to Performance and Physiological Responses Following a Supramaximal Judo Test? PLoS ONE, 2013; 8: $1-11$ 
Boguszewska K, Boguszewski D, Buśko K. Special judo fitness test and biomechanics measurements as a way to control of physical fitness in young judoists. Arc Budo, 2010; 6: 205-209

Brar TK, Singh KD, Kumar A. Effect of Different Phases of Menstrual Cycle on Heart Rate Variability (HRV). J. Clin. diagnostic Res, 2015; 9: 1-4

Buchheit M, Gindre C. Cardiac parasympathetic regulation: respective associations with cardiorespiratory fitness and training load. Am J Physiol Heart Circ, 2006; 291: H451-H458

Buchheit M, Laursen PB, Ahmaidi S. Parasympathetic reactivation after repeated sprint exercise. Am J Physiol Heart Circ, 2007; 293: H133-H141

Cataldo A, Daniele Z, Cerasola D, Vallone V, Russo G, Lo Presti R, Traina M. Influences of baseline heart rate variability on repeated sprint performance in young soccer players. J Sports Med Phys Fitness, 2016; 56: 491-6

Cohen, J. Statistical Power Analysis for the Behavioral Sciences. Lawrence Erlbaum (2nd ed.). Hillsdale, New York: Lawrence Erlbaum; 1988

Detanico D, Dal Pupo J, Franchini E, Santos SG. Relationship of aerobic and neuromuscular indexes with specific actions in judo. Sci Sports, 2012; 27: 16-22

Douglas J, Plews DJ, Handcock PJ, Rehrer NJ. The Beneficial Effect of Parasympathetic Reactivation on Sympathetic Drive During Simulated Rugby Sevens. Int J Sports Physiol Perform, 2016; 11: 480-488

Franchini E, Artioli GG, Brito CJ. Judo combat: time-motion analysis and physiology. Int J Perform Anal Sport, 2013; 13: 624-641

Franchini E, Bertuzzi RC, Takito MY, Kiss MAPDM. Effects of recovery type after a judo match on blood lactate and performance in specific and non-specific judo tasks. Eur J Appl Physiol, 2009; 107: 377-383

Franchini E, Del Vecchio FB, Matsushigue KA, Artioli GG. Physiological profiles of elite judo athletes. Sports Med, 2011a; 41: 147-166

Franchini E, Del Vecchio FB, Sterkowicz S. A special judo fitness test classificatory table. Arch Budo, 2009; 5: 127-129

Franchini E, Nunes AV, Moraes JM, Del Vecchio FB. Physical fitness and anthropometrical profile of the Brazilian male judo team. J Physiol Anthropol., 2007; 26(2): 59-67. https://doi.org/10.2114/jpa2.26.59

Franchini E, Sterkowicz S, Szmatlan-Gabrys U, Gabrys T, Garnys M. Energy system contributions to the special judo fitness test. Int J Sports Physiol Perform, 2011b; 6: 334-343

Franchini E, Takito MY, Kiss MAPDM, Sterkowicz S. Physical fitness and anthropometrical differences between elite and non-elite judo players. Biol Sport, 2005; 22: 315-328

Garbouj H, Salmi MA, Haj Sassi R, Haj Yahmed M, Chamari K, Chaouachi A. Do maximal aerobic power and blood lactate concentration affect Specific Judo Fitness Test performance in female judo athletes? Biol Sport, 2016; 33: 367-372. https://doi.org/10.5604/20831862.1221890

Hansen AL, Johnsen BH, Thayer JF. Vagal influence on working memory and attention. Int J Psychophysiol, 2003; 48: 263-274

Hedelin R, Bjerle P, Henriksson-Larsen K. Heart Rate Variability in Athletes relationship with central and peripheral performance. Med Sci Sports Exerc, 2001; 33: 1394-1398

Hynynen E, Uusitalo A, Konttinen N, Rusko H. Cardiac autonomic responses to standing up and cognitive task in overtrained athletes. Int J Sports Med, 2008; 29: 552-558

Jackson AS, Pollock ML. Generalized equations for predicting body density of men. Br J Nutr, 1978; 40: 497504

Laskowski R, Wysocki K, Multan A, Haga S. Changes in cardiac structure and function among elite judoists resulting from long-term judo practice. J Sports Med Phys Fitness, 2008; 48: 366-70

Mikołajec K, Maszczyk A, Chalimoniuk M, Langfort J, Gołaś A, Zajc A. The influence of strength exercises of the lower limbs on postural stability: A possible role of the autonomic nervous system. Isokinet Exerc Sci. 2017; 25(2): 79-89 
Molina GE, Fontana KE, Porto LGG, Junqueira LF. Post-exercise heart-rate recovery correlates to resting heart-rate variability in healthy men. Clin Auton Res, 2016; 26: 415-421

Morales J, Alamo JM, Garcia-Masso X, Busca B, Lopez JL, Serra-Ano P, Gonzalez LM. Use of Heart Rate Variability in Monitoring Stres and Recovery in Judo Athletes. J Strength Cond Res, 2014; 28: 1896-1905

Morales J, Garcia V, García-Massó X, Salvá P, Escobar, Buscà B. The Use of Heart Rate Variability in Assessing Precompetitive Stress in High-Standard Judo Athletes. Int J Sports Med, 2013; 34: 144-151

Nunan D, Donovan, GAY, Jakovljevic DG, Hodges LD, Sandercock GRH, Brodie DA. Validity and Reliability of Short-Term Heart-Rate Variability from the Polar S810. Med Sci Sports Exerc, 2006; 243-250

Panissa VLG, Cal Abad, CC, Julio UF, Andreato LV, Franchini E. High-intensity intermittent exercise and its effects on heart rate variability and subsequent strength performance. Front Physiol, 2016; 7: 1-7

Reyes del Paso GA, Langewitz W, Mulder LJM, van Roon A, Duschek S. The utility of low frequency heart rate variability as an index of sympathetic cardiac tone: A review with emphasis on a reanalysis of previous studies. Psychophysiology, 2013; 50: 477-487

Sterkowicz S, Zuchowicz A, Kubica R. Levels of anaerobic and aerobic capacity indices and results for the special fitness test in judo competitors. J Hum Kinet, 1999; 2: 115-135

Tarvainen MP, Niskanen JP, Lipponen JA, Ranta-Aho PO, Karjalainen PA. Kubios HRV- heart rate variability analysis software. Computer methods and programs in biomedicine. 2014:1:210-20

Task Force of the European Society of Cardiology and The north American Society of Pacing and Electrophysiology. Heart rate variability - Standards of measurement, physiological interpretation, an clinical use. Eur Heart J, 1996; 7: 354-381

Tulppo MP, Kiviniemi AM, Hautala AJ, Kallio M, Seppänen T, Tiinanen S, Huikuri, HVR. Sympatho-vagal interaction in the recovery phase of exercise. Clin Physiol Funct Imaging, 2011: 31: 272-281

Vacher P, Nicolas M, Mourot L. Monitoring training response with heart rate variability in elite adolescent athletes: Is there a difference between judoka and swimmers? Arch Budo, 2016; 12: 35-42

\section{Corresponding author:}

\section{Eduardo Macedo Penna}

Av. dos Universitários - Jaderlândia, Castanhal - PA, 68746-360

(+5591) 3311-4600

E-mail:em.penna@yahoo.com.br 